\title{
Treatment Continuation of Asenapine or Olanzapine in Japanese Schizophrenia Patients: A Propensity Score Matched Study
}

\author{
Haruna Matsuzaki ${ }^{1}$ \\ Masakazu Hatano (iD) 1,2 \\ Miko Iwatal \\ Shigeki Yamada' \\ 'Department of Clinical Pharmacy, Fujita \\ Health University School of Medicine, \\ Toyoake, Aichi, Japan; ${ }^{2}$ Department of \\ Psychiatry, Fujita Health University \\ School of Medicine, Toyoake, Aichi, Japan
}

Correspondence: Masakazu Hatano Fujita Health University School of Medicine, I-98 Dengakugakubo, Kutsukake, Toyoake, Aichi, 470-II92, Japan

Tel +8I 562932157

Fax +8I 562934537

Email hatanomasakazu@yahoo.co.jp
Objective: Asenapine is a second-generation antipsychotic agent that is classified as a multi-acting receptor-targeted antipsychotic and is similar to olanzapine. Our study aimed to compare the treatment continuation rate and reason for discontinuation of asenapine or olanzapine in schizophrenia using real-world data.

Methods: This design was a retrospective study. The primary endpoint was Kaplan-Meier estimates of the continuation rate at six months, with the propensity score method applied to adjust for potential confounders.

Results: A total of 95 patients were analyzed in this study (asenapine, $n=46$; olanzapine, $\mathrm{n}=49$ ). Matched data were adjusted to consider six covariates (age, sex, chlorpromazine equivalent, diazepam equivalent, history of clozapine use, and history of modified electro convulsive therapy). The continuation rate at six months was $27.3 \%$ (95\% CI, 15.6-47.6) in the asenapine group and $50.8 \%(95 \% \mathrm{CI}, 34.3-75.3)$ in the olanzapine group (hazard ratio, $0.41 ; 95 \%$ CI, $0.21-0.82 ; \mathrm{P}=0.0088$ by the Log rank test) in matched data. Cases of discontinuation because of the lack of efficacy were almost as frequent for asenapine $(13.0 \%)$ as for olanzapine $(10.2 \%)$. Discontinuation due to bitter taste $(6.5 \%)$ and burden of the dosing method $(6.5 \%)$ were observed only with asenapine, whereas anticholinergic side effects such as dry mouth (4.1\%) and constipation $(2.0 \%)$ were observed only with olanzapine.

Conclusion: The low continuation rate of asenapine in real-world data may be related to specific factors such as bitter taste and burden of the dosing method.

Keywords: asenapine, olanzapine, antipsychotic agents, schizophrenia, retrospective studies, propensity score

\section{Introduction}

The mainstay of treatment for schizophrenia is pharmacotherapy with antipsychotics, and it is important to maintain and improve adherence to medication to prevent relapse. Non-adherence to antipsychotic medication has been reported to increase the risk of relapse by five times, ${ }^{1,2}$ which is a major concern for continued pharmacotherapy. In August 2009, the US Food and Drug Administration approved asenapine for the treatment of schizophrenia and bipolar I disorder. Asenapine has been shown to improve Positive and Negative Syndrome Scale (PANSS) significantly more than placebo. ${ }^{3}$ Although there is limited evidence for direct comparisons with other antipsychotics, no significant difference between asenapine and olanzapine in terms of changes to PANSS total or negative subscale scores has been 
reported thus far. ${ }^{4}$ In the largest network meta-analysis, asenapine was ranked toward the middle out of 32 antipsychotics in overall change in symptoms. ${ }^{5}$

Asenapine has a high affinity for dopamine $\left(D_{1}, D_{2}\right.$, $\left.\mathrm{D}_{3}, \mathrm{D}_{4}\right)$, serotonin $\left(5-\mathrm{HT}_{1 \mathrm{~A}}, 5-\mathrm{HT}_{1 \mathrm{~B}}, 5-\mathrm{HT}_{2 \mathrm{~A}}, 5-\mathrm{HT}_{2 \mathrm{~B}}, 5-\right.$ $\left.\mathrm{HT}_{2 \mathrm{C}}, 5-\mathrm{HT}_{5}, 5-\mathrm{HT}_{6}, 5-\mathrm{HT}_{7}\right)$, histamine $\left(\mathrm{H}_{1}, \mathrm{H}_{2}\right)$, and $\alpha-$ adrenergic $\left(\alpha_{1}, \alpha_{2 \mathrm{~A}}, \alpha_{2 \mathrm{~B}}, \alpha_{2 \mathrm{C}}\right)$ receptors and acts as an antagonist for each. ${ }^{6}$ It is classified as multi-acting receptor-targeted antipsychotic, but has no affinity for muscarinic $\mathrm{M}_{1}$ receptors compared with olanzapine and quetiapine in the same category. ${ }^{6}$ This pharmacological profile provides the advantage that asenapine has a low risk of anticholinergic side effects (constipation and dry mouth, among others). In addition, asenapine has been shown in clinical trials to cause less weight gain than olanzapine. ${ }^{7}$ However, asenapine has been reported to cause oral hypoesthesia as a specific side effect in approximately $10 \%$ of patients owing to local anesthetic activity. ${ }^{3}$ A further unique feature of asenapine is that it is the only antipsychotic designed as a sublingual tablet. This is attributable to its extensive hepatic first-pass metabolism and an oral bioavailability of $<2 \%$. ${ }^{8}$ Therefore, it is recommended to avoid eating and drinking for 10 minutes after administration; however, concern that this complicated dosing method may lead to poor medication adherence exists. Sublingual tablets are known to cause the inconvenience of oral administration. ${ }^{9}$ A questionnaire survey of asenapine in Japan revealed that $32 \%$ of patients felt burdened by the dosing methods of sublingual tablets, suggesting that for of medication decreases satisfaction. ${ }^{10}$ However, the relationship between these factors and treatment continuation has generally been rarely evaluated in detail in clinical trials. The purpose of this study was to compare the continuation rates of treatment between asenapine and olanzapine, and to determine differences in the reasons for discontinuation.

\section{Methods}

\section{Study Design}

This design was a retrospective study based on the medical records of patients at Fujita Health University Hospital. Patients who were diagnosed with schizophrenia or schizoaffective disorder according to the definitions outlined in the Diagnostic and Statistical Manual of Mental Disorders, fifth edition, and who were prescribed asenapine or olanzapine between September 2016 and December 2019 were included in the study. Patients who were prescribed asenapine or olanzapine for temporary use in case of worsening of symptoms or as an alternative drug when existing drugs could not be used due to surgery or ileus were excluded. From medical records, we extracted data on patient characteristics (age, sex, and treatment history) and clinical course. The total dose of each antipsychotic and benzodiazepine was calculated by converting the dose to chlorpromazine and diazepam equivalents, respectively. ${ }^{11}$ Clozapine or modified electro convulsive therapy history was assessed that because treatment continuation rates may be underestimated in treatmentresistant schizophrenia.

The primary endpoint was the continuation rate of treatment at six months. The secondary endpoint was the reason for discontinuation of each antipsychotic. The reasons for discontinuation were categorized as "within 1 month" or "1-6 months."

Study procedures were performed in accordance with the principles of the Declaration of Helsinki, and this study was approved by the Institutional Review Board of Fujita Health University (HM19-050). Patients had the right to revoke their consent and opt out from the research.

\section{Statistical Analysis}

We used propensity score matching to adjust for potential confounders. The propensity score was estimated through a logistic regression model that considered six covariates (age, sex, chlorpromazine equivalent, diazepam equivalent, history of clozapine use, and history of modified electro convulsive therapy). We performed a nearest-neighbor matching algorithm without replacement using a caliper width equal to 0.2 of the standard deviation of the logit of the propensity score. We matched asenapine group and olanzapine group in a 1:1 ratio according to these covariates. The balance of the covariates between asenapine and olanzapine was checked using the standardized mean difference (SMD), with $<10 \%$ being considered well balanced. The continuation rate of treatment with asenapine or olanzapine was calculated by the Kaplan-Meier method, and the Log rank test was performed to evaluate differences between the two groups. The Cox proportional hazards model was used to estimate the hazard ratio and $95 \%$ confidence interval for asenapine as compared with that for olanzapine). $P$-values were two-sided, and those less than 0.05 were considered significant. The sample size was determined from the number of subjects enrolled within the study period. All statistical analyses were performed with $\mathrm{R} \quad 3.4 .3$ (The R Foundation for Statistical Computing). 


\section{Results}

\section{Patient Characteristics}

Data on 99 patients who were asenapine or olanzapine were extracted from medical records, and four patients were excluded from the study owing to temporary use. Thus, 95 patients were analyzed in this study, of which 46 were in the asenapine group (mean age \pm standard deviation [SD], 42.0 \pm 15.3 ; males, 18 ) and 49 were in the olanzapine group (mean age $\pm \mathrm{SD}, 43.8 \pm 15.6$; males, 18). Before propensity score matching, each treatment group differed in all characteristics except sex. After propensity score matching (33 asenapine cases matched with 33 olanzapine cases), the SMD were less than $10 \%$ for all covariates. Thus, the covariate balance in the matched data was well adjusted. The patient characteristics at baseline are summarized in Table 1.

\section{Treatment Continuation Rate}

The continuation rates of treatment as calculated by the Kaplan-Meier method are shown in Figure 1 (before propensity score matching) and Figure 2 (after propensity score matching). Before propensity score matching, the continuation rate at six months as calculated by the Kaplan-Meier method was $23.9 \%$ (95\% CI, 14.3-40.0) for the asenapine group and $51.7 \%$ (95\% CI, 37.7-71.1) for the olanzapine group (hazard ratio, $0.39 ; 95 \% \mathrm{CI}, 0.22-0.68 ; P=0.00063$ by the $\log$ rank test) (Figure 1). After propensity score matching, the continuation rate at six months was $27.3 \%(95 \% \mathrm{CI}, 15.6-47.6)$ for the asenapine group and $50.8 \%(95 \% \mathrm{CI}, 34.3-75.3)$ for the olanzapine group (hazard ratio, $0.41 ; 95 \% \mathrm{CI}, 0.21-0.82$; $P=0.0088$ by the Log rank test) (Figure 2).

\section{Reasons for Discontinuation}

Details of the reasons for discontinuation of each antipsychotic are shown in Table 2. The reasons for discontinuation observed in $>10 \%$ of the patients were lack of efficacy (13.0\%, 6/46 patients) and insomnia (10.9\%, 5/46 patients) for asenapine, and weight gain (14.3\%, $7 / 49$ patients) and lack of efficacy $(10.2 \%, 5 / 49$ patients) for olanzapine. When discontinuation was limited to within one month, asenapine was most frequently discontinued due to akathisia $(8.7 \%, 4 / 46$ patients). Olanzapine was correlated with the highest weight gain within 1 month $(6.1 \%, 3 / 49$ patients) and between 1-6 months (8.2\%, 4/49 patients). Discontinuations due to bitter taste $(6.5 \%, 3 / 46$ patients) and burden of the dosing method $(6.5 \%, 3 / 46$ patients) were observed only in asenapine cases, whereas anticholinergic side effects such as dry mouth $(4.1 \%, 2 / 49$ patients $)$ and constipation $(2.0 \%, 1 / 49$ patients) were observed only in olanzapine cases. Discontinuation owing to weight gain was observed in only one of the asenapine cases.

\section{Discussion}

This study evaluated the continuation rates of treatment and reasons for discontinuation of asenapine and olanzapine using real-world data. Asenapine had a significantly lower continuation rate than olanzapine. In addition, the same result was shown in analysis using propensity score matching to account for age, sex, antipsychotic and concomitant medication dose, and treatment history. A previous meta-analysis reported that there was no difference between asenapine and olanzapine in the rate of treatment discontinuation due to adverse events or lack of efficacy, but the overall rate of treatment continuation was significantly lower for asenapine. ${ }^{4}$ Although the cause of this difference was not discussed in detail, our findings suggest that the distinct differences in reasons for discontinuation of asenapine compared to olanzapine are relevant. In other words, factors for discontinuation of asenapine treatment do not fall under the general efficacy and safety assessment.

Cases of discontinuation because of the lack of efficacy were almost as frequent for asenapine as for olanzapine, which supported the results reported in a previous meta-analysis. ${ }^{4}$ Therefore, bitter taste and burden of the dosing method were reasons for discontinuation observed only in asenapine cases, and these factors may contribute to the lower continuation rate for asenapine. Asenapine has a local anesthetic activity and is known to cause oral hypoesthesia, ${ }^{12}$ which generally resolves within one hour ${ }^{13}$ but might affect patient adherence to medication. Although the incidence of oral hypoesthesia was reported in only $\sim 10 \%$ of cases studied in a previous randomized controlled trial, ${ }^{3}$ spontaneous reports using open questions of healthy male subjects have shown an incidence of oral paresthesia in more than $20 \%$ of cases, which was as high as $75 \%$ when mild oral paresthesia was included. ${ }^{14}$ A questionnaire survey on the experience of oral hypoesthesia when taking asenapine among schizophrenic patients in Japan similarly revealed that $\sim 70 \%$ of patients experienced a bitter taste after asenapine administration. ${ }^{10}$ These data indicate that oral hypoesthesia occurs in the majority of patients, yet it is likely to be latent in real-world practice. Although these symptoms are generally perceived to be mild, our 
Table I Patient Characteristics

\begin{tabular}{|c|c|c|c|c|c|c|}
\hline & \multicolumn{3}{|c|}{$\begin{array}{l}\text { All Cases Before Propensity Score } \\
\text { Matching }\end{array}$} & \multicolumn{3}{|c|}{$\begin{array}{l}\text { Matched Cases After Propensity Score } \\
\text { Matching }\end{array}$} \\
\hline & $\begin{array}{l}\text { Asenapine } \\
(N=46)\end{array}$ & $\begin{array}{l}\text { Olanzapine } \\
(N=49)\end{array}$ & SMD & $\begin{array}{l}\text { Asenapine } \\
(\mathrm{N}=33)\end{array}$ & $\begin{array}{l}\text { Olanzpine } \\
(N=33)\end{array}$ & SMD \\
\hline Age (year), mean \pm SD & $42.0 \pm 15.3$ & $43.8 \pm 15.6$ & 0.12 & $41.8 \pm 15.9$ & $41.4 \pm 15.3$ & 0.025 \\
\hline Male sex (\%) & $18(39.1)$ & $18(36.7)$ & 0.049 & $13(39.4)$ & $14(42.4)$ & 0.062 \\
\hline Chlorpromazine equivalent $(\mathrm{mg})$, mean $\pm \mathrm{SD}$ & $452.9 \pm 324.4$ & $500.9 \pm 396.7$ & 0.13 & $453.5 \pm 285.1$ & $437.1 \pm 342.5$ & 0.052 \\
\hline Diazepam equivalent $(\mathrm{mg})$, mean $\pm \mathrm{SD}$ & $7.7 \pm 10.8$ & $4.6 \pm 6.6$ & 0.35 & $4.8 \pm 5.8$ & $4.9 \pm 7.5$ & 0.024 \\
\hline History of clozapine use (\%) & $3(6.5)$ & $0(0.0)$ & 0.37 & $0(0.0)$ & $0(0.0)$ & $<0.001$ \\
\hline History of modified Electro Convulsive Therapy (\%) & $9(19.6)$ & $13(26.5)$ & 0.17 & $5(15.2)$ & $6(18.2)$ & 0.081 \\
\hline
\end{tabular}

Abbreviations: SD, standard deviation; SMD, standardized mean difference.

results suggest that oral hypoesthesia can cause patients to discontinue the medication. Moreover, all three patients who complained of bitter taste discontinued asenapine within a month, which indicates that bitter taste is one of the main reasons for early discontinuation. Therefore, informing patients about these potential side effects before they take asenapine is necessary.
Another characteristic reason for the discontinuation of asenapine is burden of the dosing method. Asenapine is the only antipsychotic that has been developed as a sublingual tablet, which requires the patient to avoid eating and drinking for 10 minutes after administration. A survey on the formulations of antipsychotics showed that $22.2 \%$ of patients complained about the dosing method. ${ }^{15}$ As with

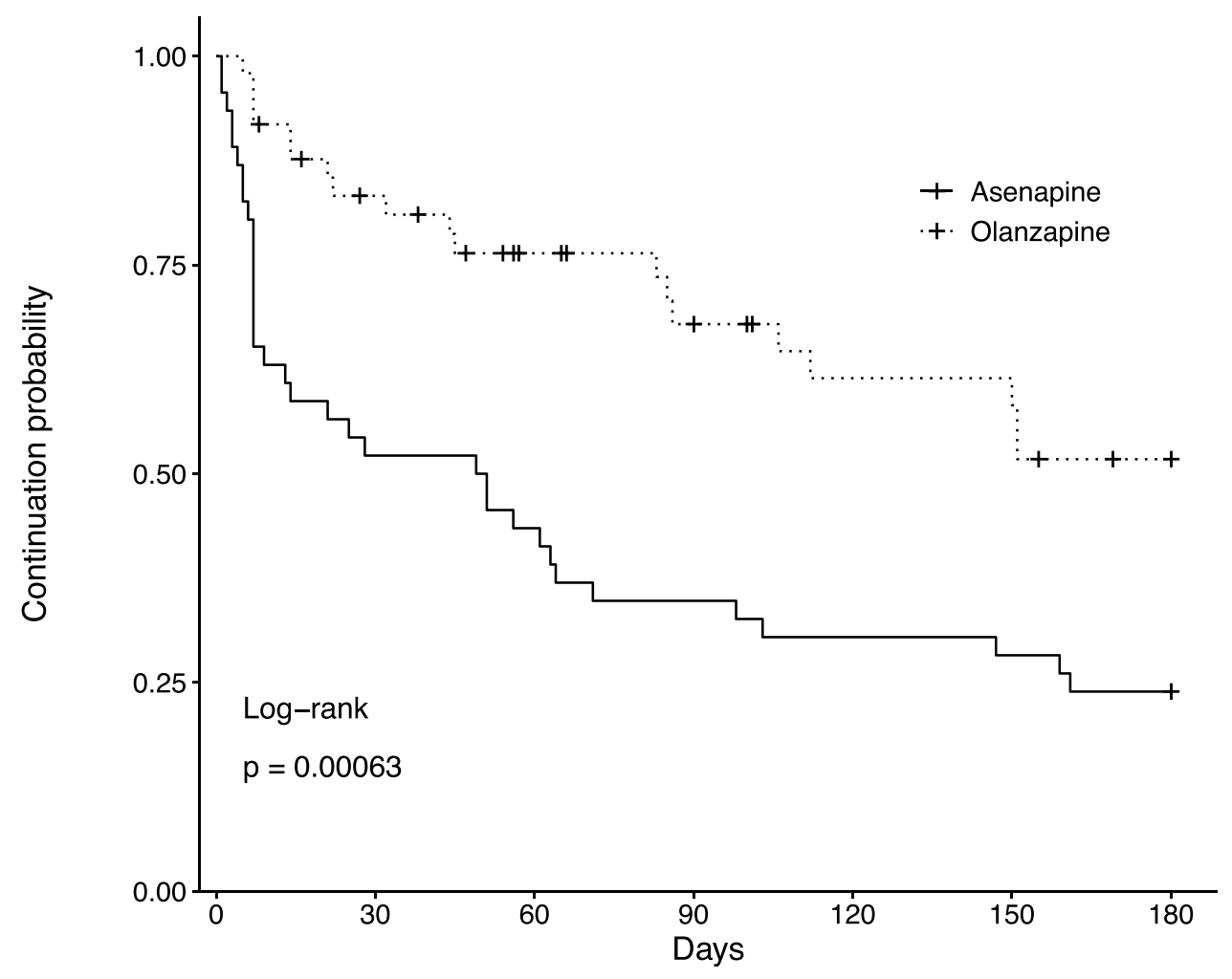

Number at risk

$\begin{array}{lllllll}\text { Asenapine } 46 & 24 & 20 & 16 & 14 & 13 & 11 \\ \text { Olanzapine } 49 & 37 & 29 & 24 & 19 & 19 & 14\end{array}$

Figure I Continuation rate of treatment calculated by the Kaplan-Meier method before propensity score matching. 
oral hypoesthesia, the burden of the dosing method is not directly associated with psychopathological mechanisms but is an important factor affecting patients' adherence to medication. Furthermore, non-compliance with this dosing method can cause a lack of elevated blood concentrations, which may be misinterpreted as insufficient efficacy and lead to discontinuation.

In contrast, the main reasons for discontinuation of olanzapine were weight gain and anticholinergic side effects, which were rarely observed with asenapine. Compared to asenapine, olanzapine has been associated with a significantly higher risk of weight gain, and our results support this finding. ${ }^{5}$ Four out of seven patients in the present study experienced weight gain and discontinued use after one month of treatment. Continuous monitoring of patients is recommended, even in the maintenance phase. In terms of anticholinergic side effects, although constipation and dry mouth were the only reasons for discontinuation of olanzapine, a variety of other symptoms, such as dysuria and cognitive impairment, might occur in real-world practice. ${ }^{16}$ It should also be noted that these anticholinergic symptoms might appear after long-term use of more than 6 months.

Our study has several limitations. We used the propensity score method to control for potential confounders but were only able to adjust for six covariates in total, including age, sex, antipsychotic and concomitant medication dose, and treatment history. Other confounders, such as psychiatric symptoms, co-morbid medical/physical health disorders, and concomitant medications (non-psychiatric), could not be considered owing to the lack of information in the medical records. Furthermore, the present study revealed the difference in reasons for discontinuation of asenapine compared with olanzapine, but the data were only numerically compared and not statistically analyzed due to the small sample size. Therefore, these factors must be analyzed using a larger data set.

In conclusion, real-world data suggest that asenapine has a significantly lower treatment continuation rate than olanzapine. Part of this difference may be

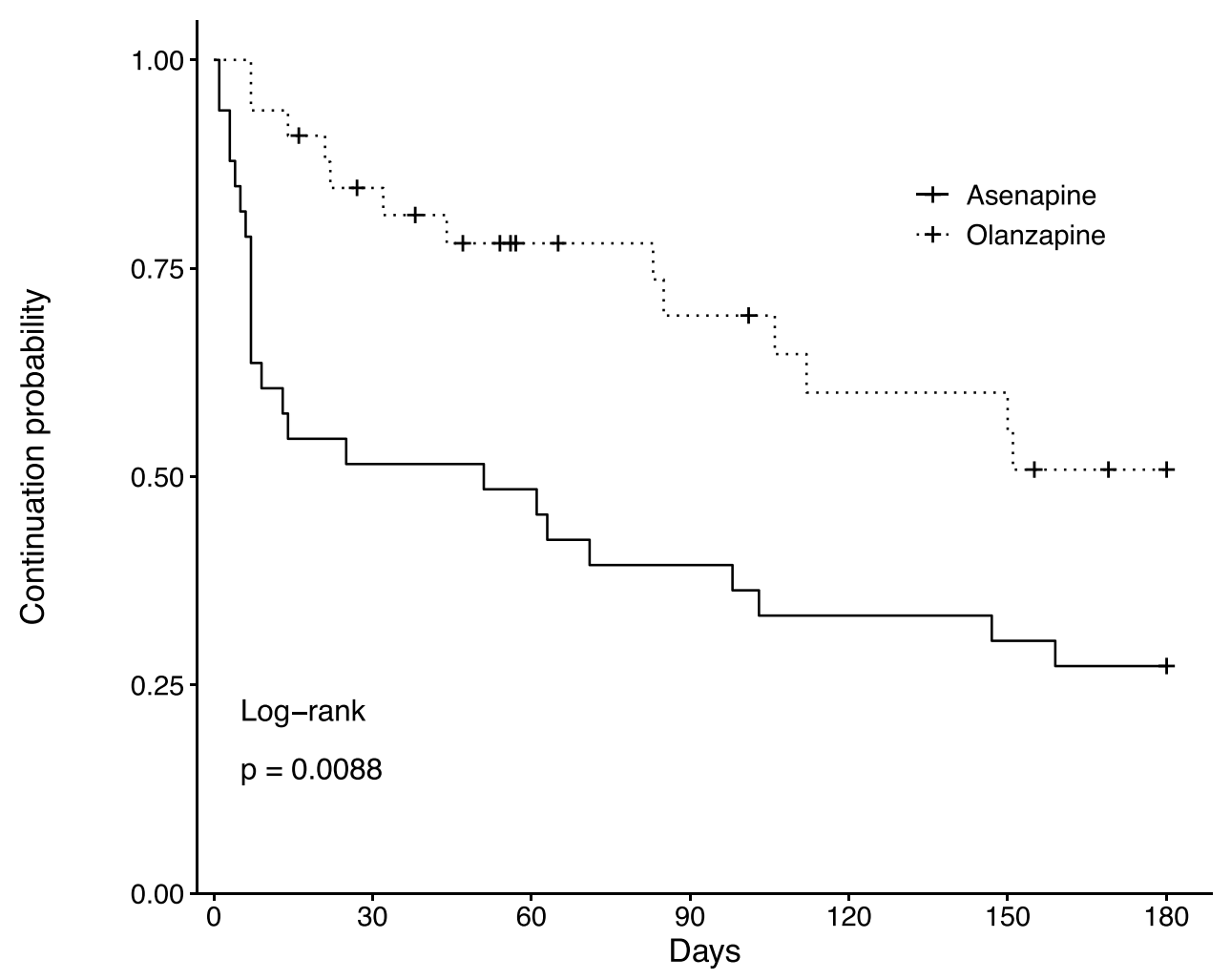

Number at risk

$\begin{array}{lllllll}\text { Asenapine 33 } & 17 & 16 & 13 & 11 & 10 & 9 \\ \text { Olanzapine 33 } & 26 & 19 & 16 & 13 & 13 & 9\end{array}$

Figure 2 Continuation rate of treatment calculated by the Kaplan-Meier method after propensity score matching. 
Table 2 Reason for Discontinuation of Each Antipsychotic

\begin{tabular}{|c|c|c|c|c|c|c|c|c|c|c|c|c|}
\hline \multirow{3}{*}{$\begin{array}{l}\text { Rseason for Discontinuation, } \mathbf{n}(\%) \\
\text { Lack of efficacy }\end{array}$} & \multicolumn{6}{|c|}{ Asenapine $(n=46)$} & \multicolumn{6}{|c|}{ Olanzapine $(n=49)$} \\
\hline & \multicolumn{2}{|c|}{ Total } & \multicolumn{2}{|c|}{$<$ I Month } & \multicolumn{2}{|c|}{ I-6 Month } & \multicolumn{2}{|c|}{ Total } & \multicolumn{2}{|c|}{$<$ I Month } & \multicolumn{2}{|c|}{ I-6 Month } \\
\hline & 6 & $(13.0)$ & 2 & $(4.3)$ & 4 & $(8.7)$ & 5 & $(10.2)$ & 2 & (4.I) & 3 & $(6.1)$ \\
\hline Insomnia & 5 & $(10.9)$ & I & $(2.2)$ & 4 & (8.7) & I & $(2.0)$ & I & $(2.0)$ & 0 & $(0.0)$ \\
\hline Akathisia & 4 & $(8.7)$ & 4 & $(8.7)$ & 0 & $(0.0)$ & 1 & $(2.0)$ & I & $(2.0)$ & 0 & $(0.0)$ \\
\hline Bitter taste & 3 & $(6.5)$ & 3 & $(6.5)$ & 0 & $(0.0)$ & 0 & $(0.0)$ & 0 & $(0.0)$ & 0 & $(0.0)$ \\
\hline Burden of dosing method & 3 & $(6.5)$ & 2 & $(4.3)$ & I & $(2.2)$ & 0 & $(0.0)$ & 0 & $(0.0)$ & 0 & $(0.0)$ \\
\hline Fatigue & 2 & $(4.3)$ & 2 & $(4.3)$ & 0 & $(0.0)$ & I & $(2.0)$ & 0 & $(0.0)$ & I & $(2.0)$ \\
\hline Dizziness & 2 & $(4.3)$ & 2 & $(4.3)$ & 0 & $(0.0)$ & 3 & $(6.1)$ & 3 & $(6.1)$ & 0 & $(0.0)$ \\
\hline Somnolence & 2 & $(4.3)$ & 2 & $(4.3)$ & 0 & $(0.0)$ & 0 & $(0.0)$ & 0 & $(0.0)$ & 0 & $(0.0)$ \\
\hline Dyspnea & 2 & $(4.3)$ & 2 & $(4.3)$ & 0 & $(0.0)$ & 0 & $(0.0)$ & 0 & $(0.0)$ & 0 & $(0.0)$ \\
\hline Dysarthria & 2 & $(4.3)$ & I & $(2.2)$ & 1 & $(2.2)$ & 0 & $(0.0)$ & 0 & $(0.0)$ & 0 & $(0.0)$ \\
\hline Weight gain & 1 & $(2.2)$ & I & $(2.2)$ & 0 & $(0.0)$ & 7 & $(14.3)$ & 3 & $(6.1)$ & 4 & $(8.2)$ \\
\hline Neuroleptic malignant syndrome & 1 & $(2.2)$ & I & $(2.2)$ & 0 & $(0.0)$ & 0 & $(0.0)$ & 0 & $(0.0)$ & 0 & $(0.0)$ \\
\hline Dystonia & 1 & $(2.2)$ & I & $(2.2)$ & 0 & $(0.0)$ & 0 & $(0.0)$ & 0 & $(0.0)$ & 0 & $(0.0)$ \\
\hline Sialorrhea & 1 & $(2.2)$ & 0 & $(0.0)$ & 1 & $(2.2)$ & 0 & $(0.0)$ & 0 & $(0.0)$ & 0 & $(0.0)$ \\
\hline Convulsion & 1 & $(2.2)$ & 1 & $(2.2)$ & 0 & $(0.0)$ & 0 & $(0.0)$ & 0 & $(0.0)$ & 0 & $(0.0)$ \\
\hline Weakness & 1 & $(2.2)$ & I & $(2.2)$ & 0 & $(0.0)$ & 0 & $(0.0)$ & 0 & $(0.0)$ & 0 & $(0.0)$ \\
\hline Increased appetite & 1 & $(2.2)$ & I & $(2.2)$ & 0 & $(0.0)$ & 0 & $(0.0)$ & 0 & $(0.0)$ & 0 & $(0.0)$ \\
\hline Photophobia & 1 & $(2.2)$ & I & $(2.2)$ & 0 & $(0.0)$ & 0 & $(0.0)$ & 0 & $(0.0)$ & 0 & $(0.0)$ \\
\hline Dry mouth & 0 & $(0.0)$ & 0 & $(0.0)$ & 0 & $(0.0)$ & 2 & $(4.1)$ & I & $(2.0)$ & I & $(2.0)$ \\
\hline Constipation & 0 & $(0.0)$ & 0 & $(0.0)$ & 0 & $(0.0)$ & I & $(2.0)$ & 0 & $(0.0)$ & 0 & $(0.0)$ \\
\hline
\end{tabular}

due to the bitter taste and burden of the dosing method experienced when taking asenapine. Although these are not directly related to psychopathological mechanisms and are generally non-serious factors, they can lead to poor adherence to medication. To help ensure patients continue asenapine treatment, it is important to inform them of the side effect of bitter taste before the drug is administered, as well as confirm the dosing method regularly.

\section{Acknowledgments}

The authors would like to thank MARUZEN-YUSHODO Co., Ltd. for the English language editing.

\section{Disclosure}

The authors report no conflicts of interest in this work.

\section{References}

1. Robinson D, Woerner MG, Alvir JMJ, et al. Predictors of relapse following response from a first episode of schizophrenia or schizoaffective disorder. Arch Gen Psychiatry. 1999;56(3):241-247. doi:10.1001/archpsyc.56.3.241

2. Caseiro O, Pérez-Iglesias R, Mata I, et al. Predicting relapse after a first episode of non-affective psychosis: a three-year follow-up study. J Psychiatr Res. 2012;46(8):1099-1105. doi:10.1016/j.jpsyc hires.2012.05.001
3. Kinoshita T, Bai YM, Kim JH, Miyake M, Oshima N. Efficacy and safety of asenapine in Asian patients with an acute exacerbation of schizophrenia: a multicentre, randomized, double-blind, 6-week, placebo-controlled study. Psychopharmacology (Berl). 2016;233 (14):2663-2674. doi:10.1007/s00213-016-4295-9

4. Orr C, Deshpande S, Sawh S, Jones PM, Vasudev K. Asenapine for the treatment of psychotic disorders: a systematic review and meta-analysis. Can J Psychiatry. 2017;62(2):123-137. doi:10.1177/ 0706743716661324

5. Huhn M, Nikolakopoulou A, Schneider-Thoma J, et al. Comparative efficacy and tolerability of 32 oral antipsychotics for the acute treatment of adults with multi-episode schizophrenia: a systematic review and network meta-analysis. Lancet. 2019;394(10202):939-951. doi:10.1016/S0140-6736(19)31135-3

6. Citrome L. Asenapine review, part I: chemistry, receptor affinity profile, pharmacokinetics and metabolism. Expert Opin Drug Metab Toxicol. 2014;10(6):893-903. doi:10.1517/17425255.2014.908185

7. Kemp DE, Zhao J, Cazorla P, et al. Weight change and metabolic effects of asenapine in patients with schizophrenia and bipolar disorder. J Clin Psychiatry. 2014;75(3):238-245. doi:10.4088/ JCP. $12 \mathrm{~m} 08271$

8. Balaraman R, Gandhi H. Asenapine, a new sublingual atypical antipsychotic. J Pharmacol Pharmacother. 2010;1(1):60-61. doi:10.4103/0976-500X.64538

9. Zhou M, Derakhshanian S, Rath A, et al. Asenapine transdermal patch for the management of schizophrenia. Psychopharmacol Bull. 2020;50(4):60-82.

10. Kamei H, Kuno M, Takeuchi I, et al. A survey of schizophrenia patients' satisfaction with a second-generation antipsychotic agent, sublingual asenapine. Jpn J Clin Psychopharmacol. 2018;21 (11):1495-1506.

11. Inada $\mathrm{T}$, Inagaki A. Psychotropic dose equivalence in Japan. Psychiatry Clin Neurosci. 2015;69(8):440-447. doi:10.1111/ pcn. 12275 
12. Citrome L. Asenapine review, part II: clinical efficacy, safety and tolerability. Expert Opin Drug Saf. 2014;13(6):803-830. doi:10.1517/ 14740338.2014.908183

13. Vieta E, Montes JM. A review of asenapine in the treatment of bipolar disorder. Clin Drug Investig. 2018;38(2):87-99. doi:10.1007/s40261-017-0592-2

14. Gerrits M, De Greef R, Peeters P. Effect of absorption site on the pharmacokinetics of sublingual asenapine in healthy male subjects. Biopharm Drug Dispos. 2010;31(5-6):351-357. doi:10.1002/bdd.718
15. Hatano M, Takeuchi I, Yamashita K, et al. Satisfaction survey on antipsychotic formulations by schizophrenia patients in Japan. Clin Psychopharmacol Neurosci. 2021;19(4):610-617. doi:10.9758/ cpn.2021.19.4.610

16. Quinn TJ, Myint PK, McCleery J, Taylor-Rowan M, Stewart C. Anticholinergic burden (prognostic factor) for prediction of dementia or cognitive decline in older adults with no known cognitive syndrome. Cochrane Database Syst Rev. 2020;2020(2). doi:10.1002/14651858.cd013521

\section{Publish your work in this journal}

Neuropsychiatric Disease and Treatment is an international, peerreviewed journal of clinical therapeutics and pharmacology focusing on concise rapid reporting of clinical or pre-clinical studies on a range of neuropsychiatric and neurological disorders. This journal is indexed on PubMed Central, the 'PsycINFO' database and CAS, and is the official journal of The International Neuropsychiatric Association (INA). The manuscript management system is completely online and includes a very quick and fair peer-review system, which is all easy to use. Visit http://www.dovepress.com/testimonials.php to read real quotes from published authors. 